\title{
Curvas de abastecimiento nutrimental y concentraciones críticas nutrimentales para Pinus patula Schl. et Cham. en etapa de vivero Nutrient supply curves and critical nutrient concentrations for Pinus patula Schl. et Cham. at the nursery stage
}

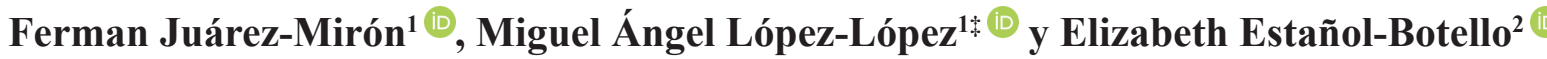 \\ ${ }^{1}$ Postgrado en Ciencias Forestales, Colegio de Postgraduados, Campus Montecillo. Carretera México-Texcoco km 36.5, Montecillo. 56230 Texcoco, Estado de \\ México, México. \\ *Autor para correspondencia (lopezma@colpos.mx) \\ ${ }^{2}$ Escuela Preparatoria Texcoco, Universidad Autónoma del Estado de México. Benjamín Aguilar Talavera Núm. 1, Fraccionamiento Las Vegas. 56180 Texcoco, \\ Estado de México, México.
}

\section{RESUMEN}

El monitoreo del estado nutrimental de la planta forestal en vivero es crucial para asegurar su calidad y aumentar la probabilidad de sobrevivencia después del trasplante. Actualmente no existen estándares nutrimentales para verificar el estado nutrimental de la producción de Pinus patula en el vivero. El objetivo del presente estudio fue generar curvas de abastecimiento nutrimental y un conjunto de concentraciones críticas foliares para plántula de esta especie. Utilizando la técnica hidropónica de subirrigación se estableció un experimento para proporcionar cinco concentraciones crecientes de nitrógeno, fósforo y potasio, para cultivar planta de $P$. patula. La fórmula de Hewitt y Smith se utilizó como solución base para implementar las concentraciones de los mencionados nutrimentos en la solución nutritiva. Se adoptó un diseño experimental completamente al azar, con cinco tratamientos por cada nutrimento estudiado. Cada tratamiento se replicó cuatro veces y la unidad experimental fue un grupo de ocho plantas. Los resultados indicaron que las variables morfológicas evaluadas se comportaron de acuerdo con un modelo sigmoidal, cuando las plantas recibieron concentraciones crecientes de un nutrimento individual. Con base en los modelos del crecimiento de las plántulas se establecieron las concentraciones de cada nutrimento en la solución nutritiva, que generan los crecimientos máximos en las distintas características morfológicas de las plantas. Se obtuvieron además las concentraciones críticas foliares de nitrógeno, fósforo, potasio, calcio, magnesio, hierro, cobre, zinc, manganeso y boro, para plántula de esta especie, cuyos valores fueron: $2.273 \%$ para $\mathrm{N}$ y 2762 , $4889,3287,981,86,4.14,13.7,437$ y $62 \mathrm{mg} \mathrm{kg}^{-1}$ para $\mathrm{P}, \mathrm{K}, \mathrm{Ca}, \mathrm{Mg}, \mathrm{Fe}, \mathrm{Cu}, \mathrm{Zn}, \mathrm{Mn}$ y B, respectivamente. El manejo nutrimental en vivero influye en la morfología de la planta, por lo que un conocimiento detallado de los efectos de cada nutrimento en sus tejidos, ayuda a producir planta prediseñada morfológicamente.

Palabras clave: concentración crítica nutrimental, hidroponía, solución nutritiva, subirrigación.

\section{SUMMARY}

Monitoring of the nutrient status of forest seedlings during the nursery stage is critical to ensure plant quality and increase its probability of survival after transplantation. At present, there are no nutritional standards that allow monitoring of the nutrient status of Pinus patula seedlings in the nursery. The objective of the present study was to derive nutrient supply curves as well as a set of foliar critical nutrient concentrations for seedlings of this species. Using the hydroponics sub-irrigation technique, an experiment was set up to provide five increasing concentrations of nitrogen, five of phosphorus, and five of potassium within

Cita recomendada:

Juárez-Mirón, F., López-López, M. Á. y Estañol-Botello, E. (2021). Curvas de abastecimiento nutrimental y concentraciones críticas nutrimentales para Pinus patula Schl. et Cham. en etapa de vivero. Terra Latinoamericana, 39, 1-14. e894. https://doi.org/10.28940/terra.v39i0.894 
the nutrient solution for growing Pinus patula seedlings. The Hewitt and Smith formula was used as the base nutrient solution to implement the concentrations of the mentioned elements in the nutrient solution. A complete randomized experimental design was adopted, which had five treatments for each nutrient studied. Each treatment was fourfold replicated, and the experimental unit was a set of eight plants. Results indicate that $P$. patula plants react according to a sigmoidal pattern to increasing concentrations of an individual nutrient. Based on the sigmoidal models for the seedlings, the concentrations of each nutrient in the nutrient solution, which maximized growth in terms of the several morphological seedling features were derived. The critical foliar concentrations of nitrogen, phosphorus, potassium, calcium, magnesium, iron, copper, zinc, manganese, and boron were also obtained for seedlings of the studied species, whose values were: $2.273 \%$ for $\mathrm{N}$, and 2762, 4889, 3287, 981, 86, 4.14, 13.7, 437, and $62 \mathrm{mg} \mathrm{kg}^{-1}$ for $\mathrm{P}, \mathrm{K}, \mathrm{Ca}, \mathrm{Mg}, \mathrm{Fe}, \mathrm{Cu}, \mathrm{Zn}, \mathrm{Mn}$, and $\mathrm{B}$, respectively. Nutrient management of seedlings in the nursery influences plant morphology, so that a detailed knowledge of the effects of each nutrient on seedlings can help produce morphologically predesigned plants.

Index words: critical nutrient concentration, hydroponics, nutrient solution, sub-irrigation.

\section{INTRODUCCIÓN}

Independientemente de las múltiples causas de la deforestación, ésta precisa de una atención puntual en todo el planeta (Geist y Lambin, 2001, 2002). Para enfrentar esta situación es importante poner en marcha programas de reforestación y de plantaciones forestales comerciales bien planeados. Sin embargo, el éxito de los programas de reforestación y plantaciones comerciales depende de múltiples factores, dentro de los que destaca la calidad de planta forestal utilizada (Mas, 2003). En efecto, la calidad con que la planta sale del vivero debe cumplir con las características morfológicas y fisiológicas apropiadas para garantizar su supervivencia después del trasplante. Se conoce como planta de calidad a aquella que reúne las características morfológicas y fisiológicas que aseguren su supervivencia y crecimiento satisfactorio bajo las condiciones ambientales y ecológicas del lugar donde serán plantadas (Prieto, García, Mejía, Huchín y Aguilar, 2009; Robles, Rodríguez y Villanueva,
2017). Las prácticas de manejo para producir plantas de calidad en un vivero, incluyen el manejo de la concentración de elementos nutricionales que necesita la planta, el espaciamiento entre riegos, la cantidad de agua por riego, el porcentaje de las mezclas de sustratos, el volumen de los envases y la densidad de planta por metro cuadrado (Rodríguez, 2010). La mayoría de factores que impactan la calidad de la planta forestal en los viveros, puede manejarse con facilidad tanto en los viveros tecnificados como en viveros hidropónicos (Rueda et al., 2014).

El estado nutrimental de la planta utilizada para la reforestación es una variable de suma importancia por la repercusión que tiene en la supervivencia y desarrollo inicial en campo (Cortina, Vilagrosa y Trubat, 2013). A pesar de lo anterior, South, Starkey y Enebak (2016), mencionan que, en el suroeste de los Estados Unidos de Norteamérica, son pocos los viveros que producen plantas en contenedor y no se tiene el seguimiento puntual acerca del estado nutrimental de las mismas. Al parecer, la falta de control del estado nutrimental de las plantas no es privativa de México, sino que ocurre incluso en los países más desarrollados como los Estados Unidos de Norteamérica. En México, con excepción de muy pocas especies de interés forestal, se desconoce los requerimientos nutrimentales de las especies durante la etapa de vivero, lo que dificulta la implementación de tecnologías para el manejo nutrimental en los viveros forestales.

Para llevar a cabo un control del estado nutrimental de la planta en el vivero es necesario contar con las herramientas técnicas necesarias, dentro de las cuales, quizá la más importante, es el conocimiento de las concentraciones críticas nutrimentales de cada una de las especies producidas. La concentración crítica de un nutrimento, en términos de porcentaje respecto a la biomasa, es aquella que permite alcanzar un rendimiento entre 90 y $100 \%$ respecto al rendimiento potencial del cultivo (Parra, López, Gómez y Ordaz, 2020), y es, tal vez, el dato más básico en términos de nutrición, si se pretende producir planta de buena calidad. La concentración crítica determinada para una especie vegetal puede variar, dependiendo de las condiciones en que se haya realizado el estudio, como lo consignan Louw y Scholes (2003), quienes afirman que una variedad de factores del sitio se correlaciona significativamente con los niveles de nutrimentos foliares, incluidos los parámetros climáticos, los niveles de cationes de la capa superior del suelo, la tasa de 
mineralización de $\mathrm{N}$ y la textura del suelo. No obstante, el uso de las concentraciones críticas para una especie sigue siendo de gran utilidad para su diagnóstico y manejo nutrimental (Landis Haase y Dumroese, 2005).

La hidroponía es el término que describe las distintas formas en las que pueden cultivarse plantas sin suelo. Los métodos hidropónicos, incluyen el cultivo de plantas en recipientes llenos de solución y cualquier otro medio distinto al suelo, incluso arena gruesa, vermiculita y otros más exóticos, como piedras compactadas, ladrillos, fragmentos de hormigón, entre otros (Andreau, Giménez y Beltrano, 2015).

Las técnicas hidropónicas son de gran utilidad para conducir estudios de nutrición en viveros forestales. Consecuentemente, el presente estudio tuvo como objetivo determinar las concentraciones óptimas requeridas, de los tres macronutrimentos primarios en la solución nutritiva; además de generar un conjunto de concentraciones críticas foliares de nitrógeno $(\mathrm{N})$, fósforo $(\mathrm{P})$, potasio $(\mathrm{K})$, calcio $(\mathrm{Ca})$, magnesio $(\mathrm{Mg})$, hierro $(\mathrm{Fe})$, cobre $(\mathrm{Cu})$, zinc $(\mathrm{Zn})$, manganeso $(\mathrm{Mn}) \mathrm{y}$ boro (B), para Pinus patula Schl. et Cham., durante su etapa de vivero.

\section{MATERIALES Y MÉTODOS}

El experimento se realizó entre el 24 de octubre del 2017 y el 15 de junio del 2018, en el ejido Palo Bendito, municipio de Huayacocotla; sitio ubicado en la zona norte del estado de Veracruz, a $20^{\circ} 27 \mathrm{~N}$ y $98^{\circ}$ 29 O, a una altitud de $2460 \mathrm{~m}$ (Figura 1). La zona tiene un clima templado subhúmedo, con lluvias en verano y nieblas frecuentes, con una temperatura anual de 3 a $8{ }^{\circ} \mathrm{C}$ en periodo de invierno y de $16{ }^{\circ} \mathrm{C}$ en las demás estaciones (Vásquez et al., 2015). El experimento se instaló en un invernadero ubicado en un claro dentro de un bosque de Pinus patula, por lo que recibe sombras de las copas de los árboles especialmente por las tardes y durante la estación de invierno.

Se utilizó un diseño experimental completamente al azar en el que se probaron 15 tratamientos correspondientes a tres series. La serie uno correspondió a cinco concentraciones de $\mathrm{N}$ en la solución nutritiva; la serie dos correspondió a cinco concentraciones de $\mathrm{P}$, y la serie tres, a cinco de K. Los niveles en la primera serie fueron $0,75,150,225$ y $300 \mathrm{mg} \mathrm{L}^{-1} \mathrm{de} \mathrm{N}$; los de la segunda fueron: $0,50,100,150$ y $200 \mathrm{mg} \mathrm{L}^{-1} \mathrm{de} \mathrm{P} \mathrm{y}$

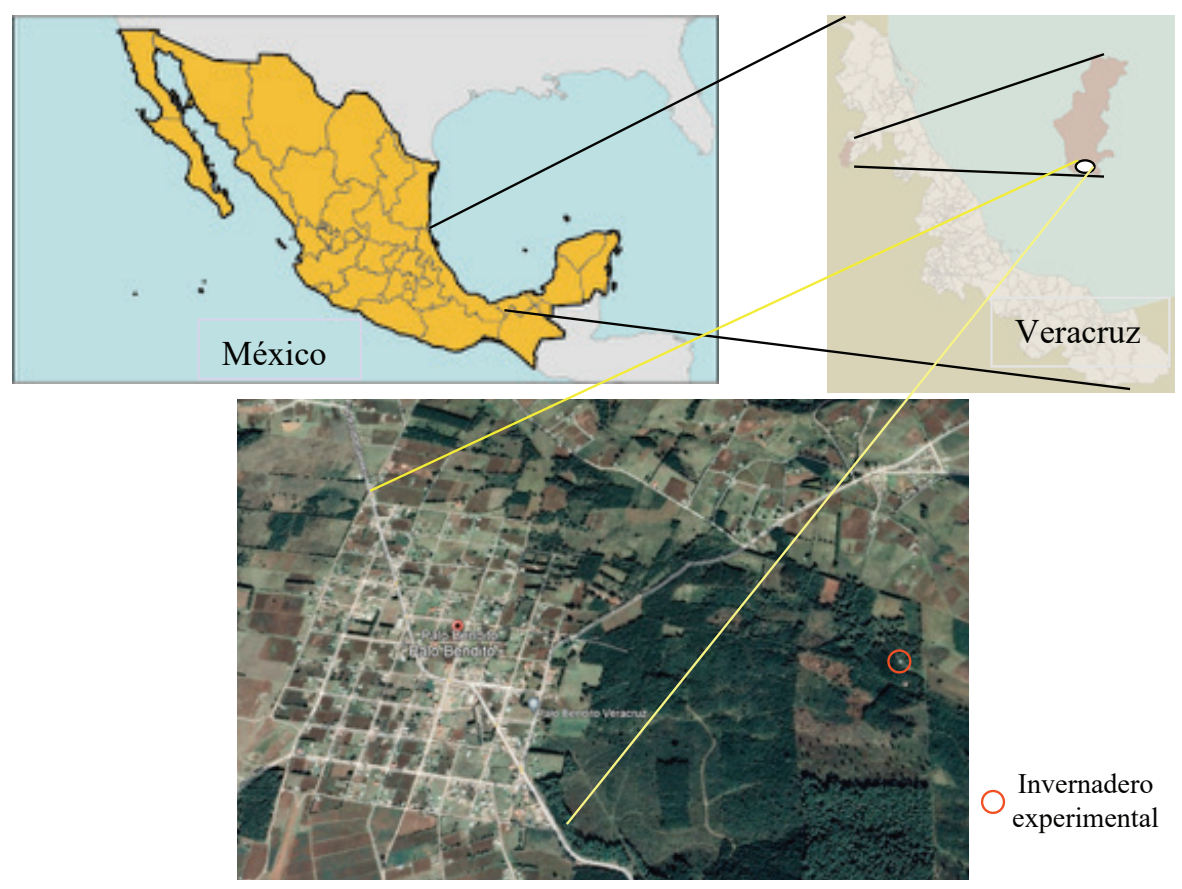

Figura 1. Área de estudio del experimento de concentraciones críticas nutrimentales de Pinus patula, realizado en Huayacocotla, Veracruz, México.

Figure 1. Study area for the experiment on critical nutrient concentrations of Pinus patula, carried out at Huayacocotla, Veracruz, Mexico. 
los de la tercera fueron: $0,80,160,240$ y $320 \mathrm{mg} \mathrm{L}^{-1}$ de K. Cada tratamiento constó en cuatro repeticiones y cada unidad experimental estuvo constituida por ocho plantas. Los tratamientos probados se enlistan en el Cuadro 1.

Las plantas crecieron en tubetes de color negro de $400 \mathrm{~cm}^{3}, 4.5 \mathrm{~cm}$ de diámetro superior y $17 \mathrm{~cm}$ de longitud (Figura 2). Se utilizó como sustrato tezontle rojo cuyo diámetro varió de $0.5 \mathrm{a} 1 \mathrm{~cm}$. El abastecimiento de agua y nutrimentos (solución nutritiva) se realizó diariamente en forma manual utilizando un sistema de subirrigación, que consistió en la inmersión de cada unidad experimental en el recipiente que contenía su correspondiente solución nutritiva. Después de la inmersión, las unidades experimentales se dejaron drenar sobre el recipiente de la solución nutritiva correspondiente, misma que se renovó cada 15 días. La aplicación de las soluciones nutritivas inició a los 15 días de trasplantada la plántula en el tubete (1 de enero del 2018).

Cuadro 1. Tratamientos probados en el experimento de nutrición de Pinus patula Schl. et Cham., en etapa de vivero, en Huayacocotla, Veracruz, México.

Table 1. Treatments tested in the Pinus patula Schl. et Cham. nursery nutrition experiment, at Huayacocotla, Veracruz, Mexico.

\begin{tabular}{|c|c|c|c|c|}
\hline \multirow{2}{*}{ Tratamiento } & \multirow{2}{*}{ Serie } & \multicolumn{3}{|c|}{ Concentración en la solución nutritiva } \\
\hline & & $\mathrm{N}$ & $\mathrm{P}$ & $\mathrm{K}$ \\
\hline & & $-\cdots$ & $\mathrm{mg} \mathrm{L}^{-1}$ & --- \\
\hline $\mathrm{T} 1$ & 1 & 0 & 41 & 156 \\
\hline $\mathrm{T} 2$ & 1 & 75 & 41 & 156 \\
\hline $\mathrm{T} 3$ & 1 & 150 & 41 & 156 \\
\hline $\mathrm{T} 4$ & 1 & 225 & 41 & 156 \\
\hline T5 & 1 & 300 & 41 & 156 \\
\hline T6 & 2 & 170 & 0 & 156 \\
\hline $\mathrm{T} 7$ & 2 & 170 & 50 & 156 \\
\hline $\mathrm{T} 8$ & 2 & 170 & 100 & 156 \\
\hline T9 & 2 & 170 & 150 & 156 \\
\hline T10 & 2 & 170 & 200 & 156 \\
\hline T11 & 3 & 170 & 41 & 0 \\
\hline T12 & 3 & 170 & 41 & 80 \\
\hline T13 & 3 & 170 & 41 & 160 \\
\hline T14 & 3 & 170 & 41 & 240 \\
\hline $\mathrm{T} 15$ & 3 & 170 & 41 & 320 \\
\hline
\end{tabular}

La fórmula base de solución nutritiva utilizada en este trabajo fue la solución tipo amonio de Hewitt y Smith (1974) (Cuadro 2), la cual fue modificada para ajustarla a cada uno de los tratamientos a probar. La fórmula se ajustó de acuerdo con las concentraciones de N, P y K planteadas en el experimento, mientras los nutrimentos restantes permanecieron en las concentraciones indicadas por Hewitt y Smith (1974). En el caso del azufre las concentraciones variaron en todos los tratamientos, ya que el azufre forma parte de varios materiales fertilizantes y es difícil mantener la concentración indicada en la fórmula.

El pH de la solución nutritiva se midió diariamente y se ajustó a 5. Con este fin se utilizó hidróxido de sodio para aumentarlo y ácido sulfúrico para disminuirlo. La medición del $\mathrm{pH}$ se hizo utilizando un potenciómetro de la marca HANNA modelo HI98130.

Todas las plantas se midieron al final del experimento; entre el 15 y 23 de junio de 2018. La altura de las plántulas se midió con una regla metálica graduada en milímetros; para ello se consideró la distancia entre el cuello de la raíz y la yema apical de las plántulas. En el caso del diámetro, éste se midió en el cuello de la raíz, con la ayuda de un vernier digital marca Truper. Una vez que la planta fue extraída y medida la raíz, se procedió a separar la parte aérea de la raíz; la separación se realizó exactamente en el cuello de la raíz que se encontraba en la superficie del sustrato. Posteriormente, los componentes de la plántula se colocaron en bolsas de papel estraza para ser secados en una estufa marca FELISA, a una temperatura de $70{ }^{\circ} \mathrm{C}$ hasta obtener peso constante.

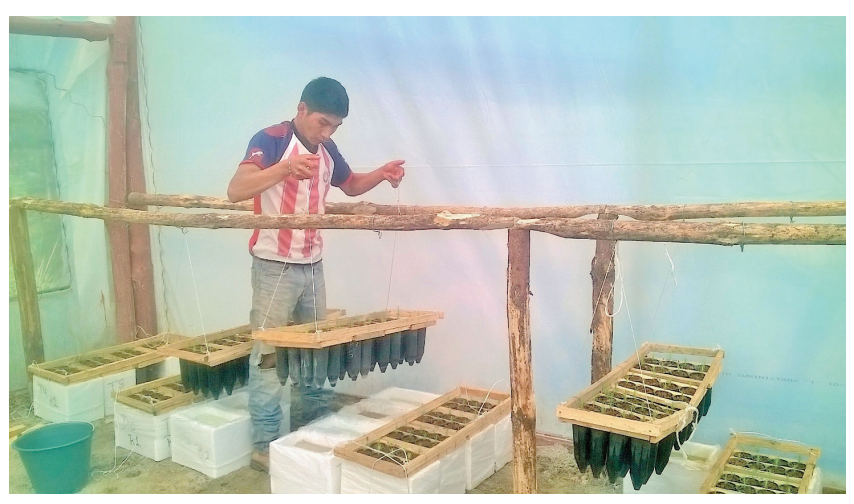

Figura 2. Vista parcial del ensayo de Pinus patula cultivado en soluciones nutritivas en Huayacocotla, Veracruz, México.

Figure 2. Partial view of the Pinus patula essay cultivated in nutrient solutions at Huayacocotla, Veracruz, Mexico. 
Cuadro 2. Concentraciones totales de nutrimentos según la fórmula de solución nutritiva de Hewitt y Smith, utilizada en el experimento de nutrición de Pinus patula Schl. et Cham, establecido en sistema hidropónico en Huayacocotla, Veracruz, México.

Table 2. Total nutrient concentrations according to the Hewitt and Smith, nutrient solution formula, used in the Pinus patula Schl. et Cham. nutrition experiment established under a hydroponic system at Huayacocotla, Veracruz, Mexico.

\begin{tabular}{lcc}
\hline \multirow{2}{*}{ Nutrimento } & \multicolumn{2}{c}{ Concentración en la solución nutritiva } \\
\cline { 2 - 3 } & Solución tipo nitratos & Solución tipo amonio \\
\hline $\mathrm{N}$ & $-\ldots-\mathrm{mg} \mathrm{L}^{-1}-\ldots$ & 113 o 141 \\
$\mathrm{P}$ & 170 & 41 \\
$\mathrm{~K}$ & 41 & 156 \\
$\mathrm{Ca}$ & 156 & 160 \\
$\mathrm{Mg}$ & 160 & 36 \\
$\mathrm{Fe}$ & 36 & 5.6 \\
$\mathrm{Mn}$ & 5.6 & 0.55 \\
$\mathrm{Zn}$ & 0.55 & 0.065 \\
$\mathrm{Cu}$ & 0.065 & 0.064 \\
$\mathrm{~B}$ & 0.064 & 0.54 \\
$\mathrm{Mo}$ & 0.54 & 0.048 \\
$\mathrm{Co}$ & 0.048 & 0.012 \\
\hline
\end{tabular}

Después se registró el peso seco de cada componente, utilizando una balanza analítica marca Ohaus con 1/1000 g de precisión. Del follaje de cada tratamiento se conformó tres muestras compuestas, a partir de varias plantas representativas. Las muestras foliares se utilizaron para determinar las concentraciones de N, P, K, Ca, Mg, Fe, Cu, Zn, Mn y B. Los análisis químicos se hicieron en el laboratorio Salvador Alcalde Blanco del Colegio de Postgraduados. El N se determinó por el método micro-Kjeldahl, mientras que los demás nutrimentos se determinaron mediante un espectrofotómetro de plasma inductivamente acoplado (ICP-AES) (VARIAN 725-ESI), a partir de un digestado obtenido a través de una mezcla de ácidos nítrico, sulfúrico y perclórico.

Para cada serie de tratamientos correspondientes a las dosis crecientes de un nutrimento en la solución nutritiva, se realizó análisis de varianza, verificando antes el cumplimiento de los supuestos de esta prueba paramétrica, así como pruebas de Tukey $(\alpha=0.05)$.
Además, para cada variable evaluada dentro de una serie de tratamientos se modeló el comportamiento de las plantas, ajustando los datos a un modelo polinómico de tercer orden. A partir de las curvas obtenidas se determinó, mediante la solución de las derivadas de las curvas, los puntos que representaron los valores máximos de cada variable de respuesta y las correspondientes concentraciones del nutrimento en la solución nutritiva.

La determinación de las concentraciones críticas se hizo mediante la metodología descrita por Parra et al. (2020), a partir de una base de datos constituida por 45 observaciones. Cada observación contuvo las concentraciones foliares de cada uno de los nutrimentos y los valores de cada una de las variables morfológicas de respuesta. La concentración crítica de un nutrimento para una variable de respuesta fue la concentración media de las tres observaciones que mostraron los valores máximos de tal variable de respuesta.

\section{RESULTADOS Y DISCUSIÓN}

\section{Curvas de Abastecimiento Nutrimental}

La Figura 3 muestra el comportamiento general de la curva de abastecimiento de nitrógeno, mismo que coincide con el de la mayoría de las variables de respuesta (Figura 4). La Figura 4 indica que el efecto

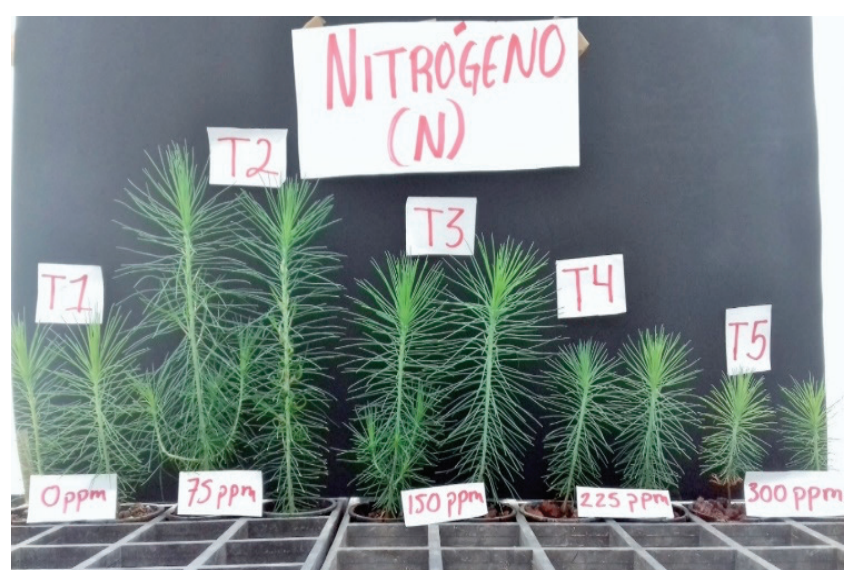

Figura 3. Comportamiento del crecimiento de altura de planta de Pinus patula de siete meses de edad, cultivada en soluciones nutritivas con concentraciones crecientes de nitrógeno $(\mathrm{N})$.

Figure 3. Behavior of height growth of seven-month old Pinus patula seedlings grown in nutrient solutions with increasing concentrations of nitrogen $(N)$. 
de las dosis crecientes de nitrógeno fue significativo, lo que expresa que las concentraciones probadas cubrieron la mayor parte de la curva de abastecimiento de este nutrimento.

Las curvas de abastecimiento de $\mathrm{N}$ de la Figura 4, indican que para las condiciones en que se desarrolló el experimento, las concentraciones de $\mathrm{N}$ que optimizan las variables estudiadas son relativamente bajas, comparadas con las indicadas por Hewitt y
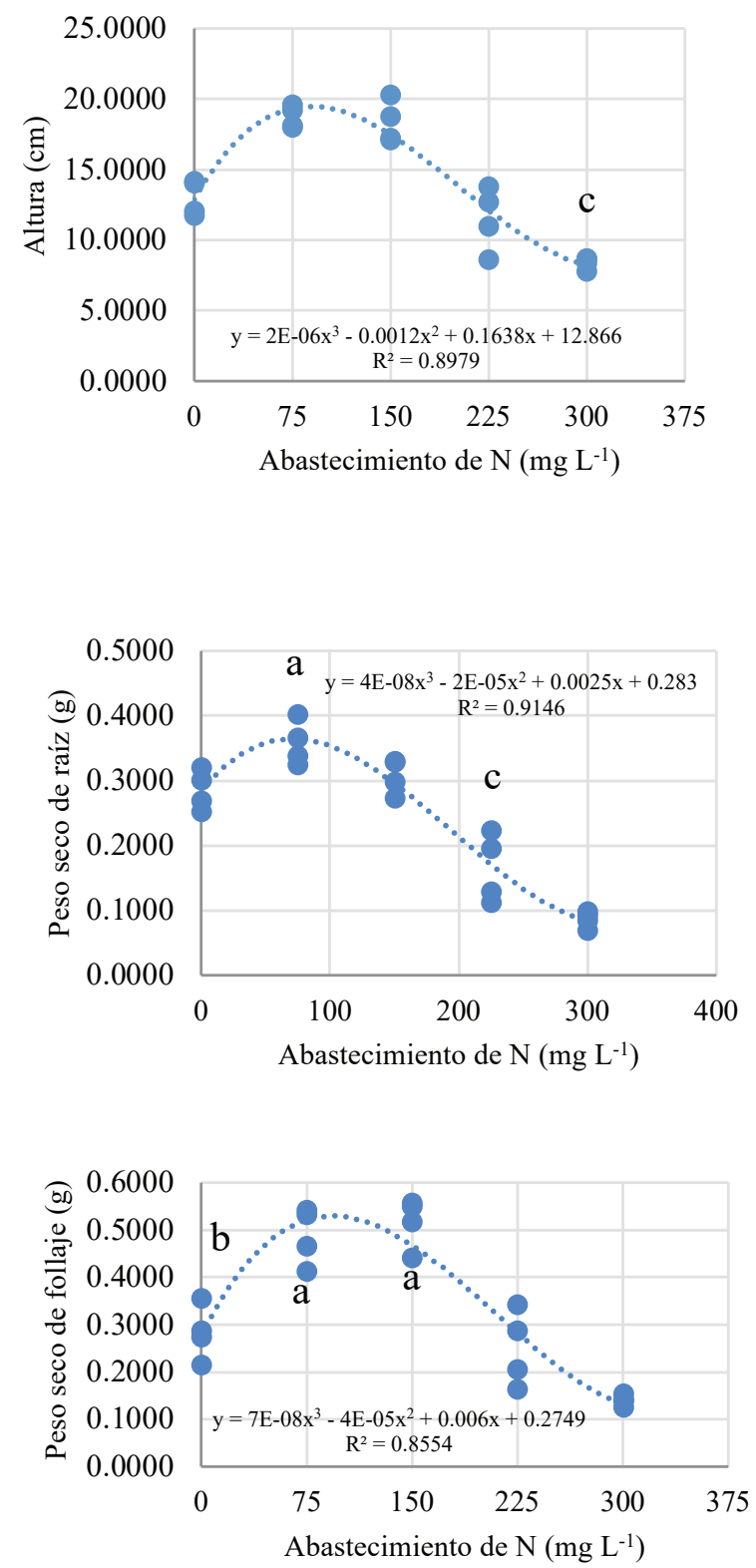

Smith (1974). En efecto, la concentración propuesta por los mencionados autores es de $170 \mathrm{mg} \mathrm{L}^{-1} \mathrm{de} \mathrm{N}$ en la solución nutritiva tipo nitratos y de 113 a 141 para la solución tipo amonio, mientras que la mayoría de las variables analizadas en el presente estudio se optimizaron con concentraciones de $\mathrm{N}$ que fluctuaron alrededor de $92 \mathrm{mg} \mathrm{L}^{-1}$ (Figura 4; Cuadro 3), con los valores específicos por variable morfológica que se indican en el mencionado cuadro.
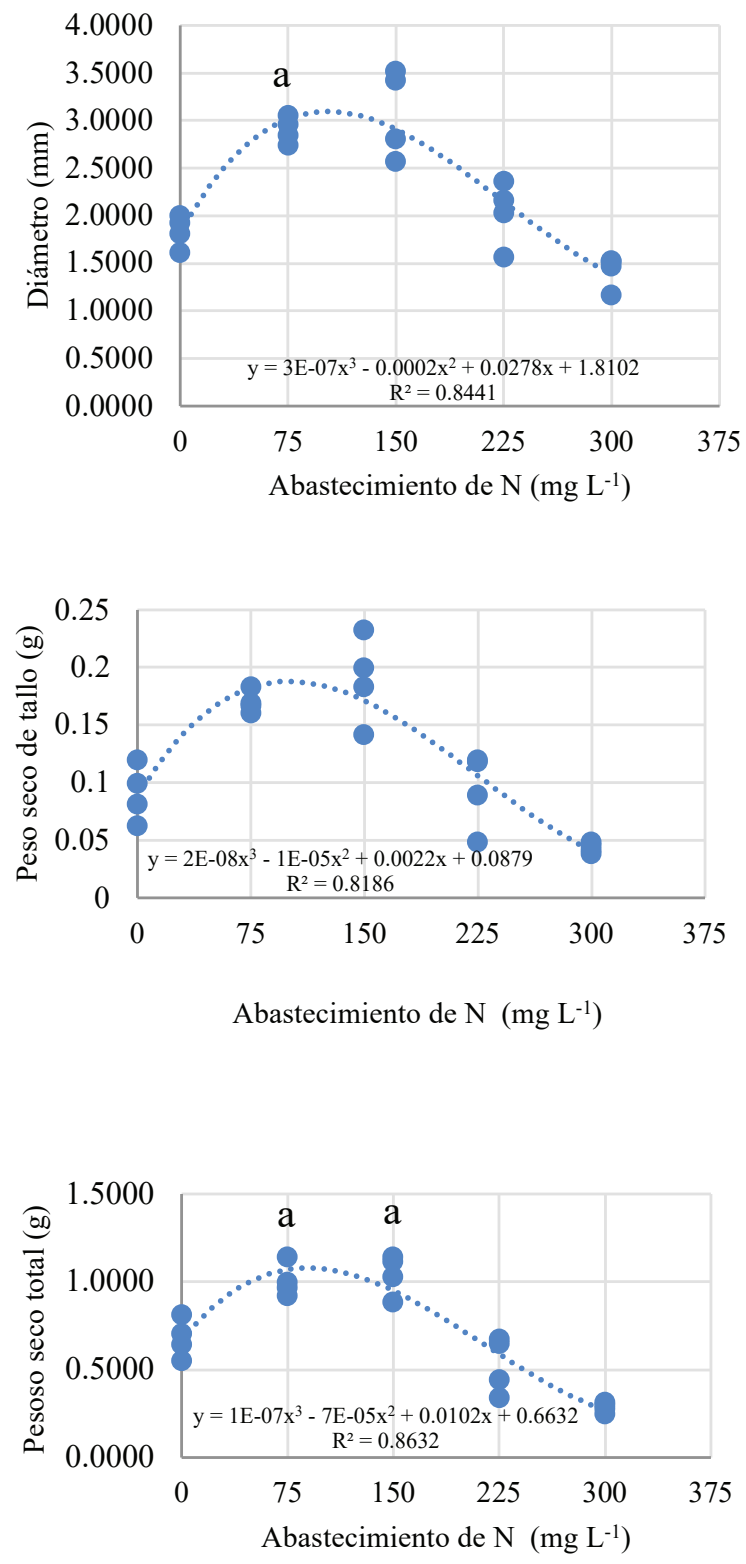

Figura 4. Curvas de abastecimiento de nitrógeno (N) para Pinus patula en etapa de vivero, cultivado en un sistema hidropónico por subirrigación.

Figure 4. Nitrogen (N) supply curves for Pinus patula at the nursery stage, grown in a subirrigation hydroponics system. 
Cuadro 3. Modificaciones propuestas a la concentración de nitrógeno en la solución nutritiva de Hewitt y Smith, para optimizar el crecimiento de diversas variables morfológicas de Pinus patula en etapa de vivero.

Table 3. Suggested changes to the nitrogen concentration in the Hewitt and Smith nutrient solution for optimizing growth of several morphological variables in Pinus patula seedlings during the nursery stage.

\begin{tabular}{|c|c|c|c|}
\hline $\begin{array}{l}\text { Variable } \\
\text { morfológica }\end{array}$ & Ecuación de abastecimiento & Primera derivada & $\operatorname{COSN}^{\dagger}$ \\
\hline Alt $(\mathrm{cm})$ & Alt $=2 \mathrm{E}-06[\mathrm{~N}]^{3}-0.0012[\mathrm{~N}]^{2}+0.1638[\mathrm{~N}]+12.866$ & $6 \mathrm{E}-06[\mathrm{~N}]^{2}-0.0024[\mathrm{~N}]+0.1638$ & 87.3 \\
\hline $\mathrm{D}(\mathrm{mm})$ & $\mathrm{D}=3 \mathrm{E}-07[\mathrm{~N}]^{3}-0.0002[\mathrm{~N}]^{2}+0.0278[\mathrm{~N}]+1.8102$ & $0.0000009[\mathrm{~N}]^{2}-0.0004[\mathrm{~N}]+0.0278$ & 86.2 \\
\hline PSFo $(g)$ & $\mathrm{PSFo}=7 \mathrm{E}-08[\mathrm{~N}]^{3}-4 \mathrm{E}-05[\mathrm{~N}]^{2}+0.006[\mathrm{~N}]+0.2749$ & $0.00000021[\mathrm{~N}]^{2}-0.00008[\mathrm{~N}]+0.006$ & 102.6 \\
\hline $\operatorname{PSTa}(\mathrm{g})$ & $\mathrm{PSTa}=0.0000000208[\mathrm{~N}]^{3}-0.0000141[\mathrm{~N}]^{2}+0.0022[\mathrm{~N}]+0.0879$ & $0.0000000208[\mathrm{~N}]^{2}-0.0000141[\mathrm{~N}]+0.0022$ & 100.3 \\
\hline $\operatorname{PSRa}(g)$ & $\mathrm{PSR}=4 \mathrm{E}-08[\mathrm{~N}]^{3}-2 \mathrm{E}-05[\mathrm{~N}]^{2}+0.0025[\mathrm{~N}]+0.283$ & $0.00000016[\mathrm{~N}]^{2}-0.00004[\mathrm{~N}]+0.0025$ & 83.3 \\
\hline PSTo $(g)$ & $\mathrm{PSTo}=1 \mathrm{E}-07[\mathrm{~N}]^{3}-7 \mathrm{E}-05[\mathrm{~N}]^{2}+0.0102[\mathrm{~N}]+0.6632$ & $0.0000003[\mathrm{~N}]^{2}-0.00014[\mathrm{~N}]+0.0102$ & 90.3 \\
\hline Media & & & 91.7 \\
\hline
\end{tabular}

Las concentraciones óptimas en la solución nutritiva son parecidas a las recomendadas por Aldana y Aguilera (2003) para especies de rápido crecimiento, como lo es Pinus patula, en su etapa de crecimiento exponencial en vivero $\left(75\right.$ y $120 \mathrm{mg} \mathrm{L}^{-1} \mathrm{~N}$ en las etapas de crecimiento inicial y exponencial, respectivamente). Sin embargo, en un estudio realizado por López-López $\left(1989^{1}\right)$, una concentración de $225 \mathrm{mg} \mathrm{L}^{-1}$ de $\mathrm{N}$ en la solución nutritiva fue insuficiente para optimizar el crecimiento de $P$. patula, abastecido con la misma solución nutritiva (Hewitt y Smith, 1974) en vivero. La diferencia entre los valores óptimos encontrados en el presente estudio y los reportados por López-López $\left(1989^{1}\right)$, pueden deberse a las condiciones ambientales en que se desarrolló ambos estudios. El estudio de López-López $\left(1989^{1}\right)$ se condujo en Montecillo, Texcoco, Edo. de México, en condiciones de un invernadero que recibía radiación solar sin restricción por todas direcciones y temperatura media de $23{ }^{\circ} \mathrm{C}$; en cambio, el presente estudio se desarrolló en un invernadero en Huayacocotla, Veracruz, en el que se registró una temperatura de 3 a $8{ }^{\circ} \mathrm{C}$ en periodo de invierno y de $16^{\circ} \mathrm{C}$ en las demás estaciones. Por otro lado, el invernadero de Huayacocotla recibió sombra de árboles cercanos, por encontrarse inserto en un bosque de pino. Ambos factores pudieron haber limitado el crecimiento de las plantas, haciendo innecesario un mayor abastecimiento de $\mathrm{N}$ en el caso del presente estudio.

Riechelman, Postma, Specken y De Haan (2021) aseguran que existen pruebas que demuestran efectos de la temperatura y radiación solar sobre las concentraciones críticas de nutrimentos como boro, pero falta información al respecto. Al existir baja radiación solar, como en el caso del presente experimento, este factor ambiental se convierte en el principal factor limitante del crecimiento. En este caso, incluso un bajo abastecimiento de nutrimentos puede ser suficiente para que la planta mantenga una concentración adecuada de ese nutrimento en sus tejidos, debido al denominado "efecto de concentración nutrimental", toda vez que la proporción de nutrimentos absorbidos puede ser elevada (aun existiendo bajas concentraciones de nutrimentos en la solución nutritiva), en relación a la cantidad de materia orgánica producida durante el proceso fotosintético, que es limitado por la escasa

\footnotetext{
${ }^{1}$ López-López, M. Á. (1989). Estudio de nutrición de Pinus patula Schl. et Cham, en sistema hidropónico. Tesis profesional. Departamento de Bosques, Universidad Autónoma Chapingo. Chapingo, México. 69 p.
} 
radiación solar (Bres y Jerzy, 2008). Este efecto de concentración puede explicar el relativamente bajo requerimiento de $\mathrm{N}$ determinado en el presente estudio.

El Cuadro 3 indica que las diversas variables morfológicas de las plantas son optimizadas con diferentes concentraciones de $\mathrm{N}$ en la solución nutritiva. Así, un crecimiento óptimo de la raíz, en términos de peso seco, se logra con sólo $83 \mathrm{mg} \mathrm{L}^{-1}$ de $\mathrm{N}$, mientras que, al parecer, si se desea optimizar la producción de follaje en las plántulas, la concentración de $\mathrm{N}$ en la solución debe aumentarse a casi $103 \mathrm{mg} \mathrm{L}^{-1}$. Este hallazgo soporta la idea de disminuir la concentración de $\mathrm{N}$ en la solución nutritiva durante la primera etapa de desarrollo de la planta en el vivero; etapa denominada de crecimiento inicial, misma que se caracteriza por el aporte de bajas concentraciones de $\mathrm{N}$ y elevadas concentraciones de $\mathrm{P}$ y a veces de $\mathrm{K}$ (Douglass $\mathrm{y}$ Landis, 2014).

De acuerdo con el cuadro mencionado arriba, el PSRa es la variable que se optimiza con la menor concentración de $\mathrm{N}$ en la solución nutritiva $\left(83.3 \mathrm{mg} \mathrm{L}^{-1}\right.$ de $\mathrm{N})$. Contrariamente, el follaje es el órgano que requiere del mayor abastecimiento de $\mathrm{N}\left(102.6 \mathrm{mg} \mathrm{L}^{-1}\right)$ si se desea optimizar su biomasa.

La Figura 5 muestra la curva de abastecimiento de P para el crecimiento de altura en plántulas de Pinus patula en vivero. Esta curva coincide con la forma general que muestran los modelos que describen el comportamiento de las diversas variables morfológicas

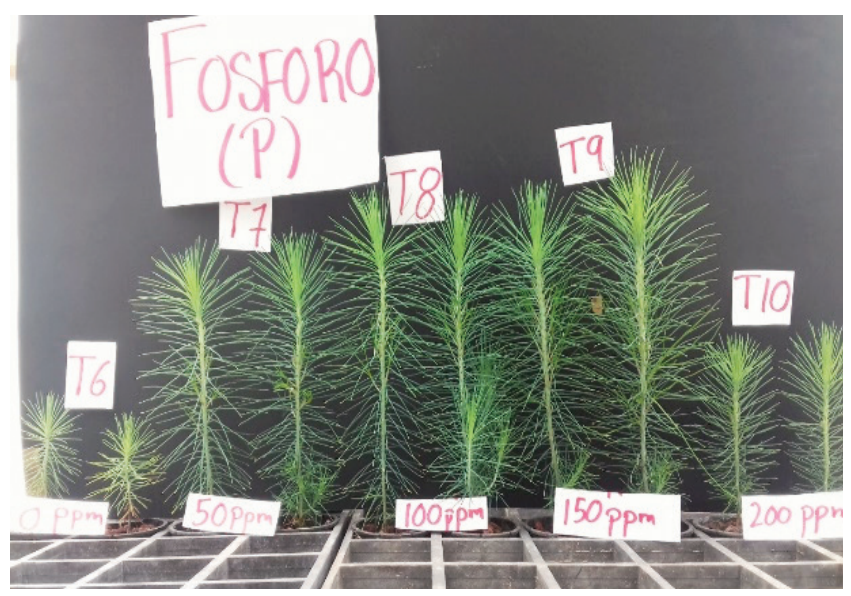

Figura 5. Comportamiento del crecimiento de altura de planta de Pinus patula en soluciones nutritivas con concentraciones crecientes de fósforo $(P)$.

Figure 5. Behavior of height growth of Pinus patula seedlings grown in nutrient solutions with increasing concentrations of phosphorus (P). al variar la concentración de $\mathrm{P}$ en la solución nutritiva de Hewitt y Smith (1974), mientras los demás nutrimentos permanecieron en la solución nutritiva en las concentraciones indicadas por los autores de la misma (Figura 6).

Como en el caso del N, la concentración óptima de $\mathrm{P}$ en la solución nutritiva varía de acuerdo con la variable morfológica a optimizar. La media de las concentraciones óptimas de $\mathrm{P}$ para todas las variables morfológicas es $123.7 \mathrm{mg} \mathrm{L}^{-1}$; concentración cercana a la encontrada por López-López $\left(1989^{1}\right)$ para la misma especie (134 $\left.\mathrm{mg} \mathrm{L}^{-1}\right)$. Se advierte que el PSRa es la variable que requiere de la mayor concentración de P (132.3 mg L-1; Cuadro 4, Figura 6) en la solución nutritiva para alcanzar valores máximos, mientras que el crecimiento de altura de plantas es la variable que requiere las menores concentraciones de este nutrimento (108.3 $\left.\mathrm{mg} \mathrm{L}^{-1}\right)$.

Los altos requerimientos de $\mathrm{P}$ por parte de la raíz de plántulas de P. patula han sido documentados por López-López $\left(1989^{1}\right)$. Este autor reportó que la única variable para la que no se alcanzó el valor crítico de $\mathrm{P}$ con las concentraciones probadas en su estudio $(0 \mathrm{a}$ $\left.150 \mathrm{mg} \mathrm{L}^{-1}\right)$ fue la longitud de raíz. González et al. (2020) indican que un adecuado abastecimiento de P a la planta resulta en un incremento en la producción de raíces nuevas. En general, el alto requerimiento de $\mathrm{P}$ por las plántulas de coníferas es ampliamente reconocido por los productores de planta en vivero, quienes usualmente incrementan la concentración de este nutrimento en la solución nutritiva durante la fase denominada "crecimiento inicial", en el proceso de producción de planta en vivero (Prieto et al., 2009).

La Figura 7 representa la curva de abastecimiento de potasio en relación con la altura de las plántulas, la cual se asemeja a las curvas de las variables morfológicas restantes (Figura 8).

Las curvas de abastecimiento de las diversas variables morfológicas estudiadas presentan la forma de una ecuación de tercer orden (Figura 8). De acuerdo con el Cuadro 5, el PSRa es la variable que presenta el mayor requerimiento de $\mathrm{K}$ en la solución nutritiva (189.7 $\mathrm{mg} \mathrm{L}^{-1}$ ), mientras que la variable que menor cantidad de K requiere es el PSTo. La concentración media de $\mathrm{K}$ en la solución nutritiva, para las variables morfológicas estudiadas es $142.9 \mathrm{mg} \mathrm{L}^{-1}$. Pareciera que esta concentración de $\mathrm{K}$ es baja; sin embargo, Massone, Bartoli y Pastorino (2018), encontraron que una concentración de $39 \mathrm{mg} \mathrm{L}^{-1}$ de $\mathrm{K}$ en la solución 

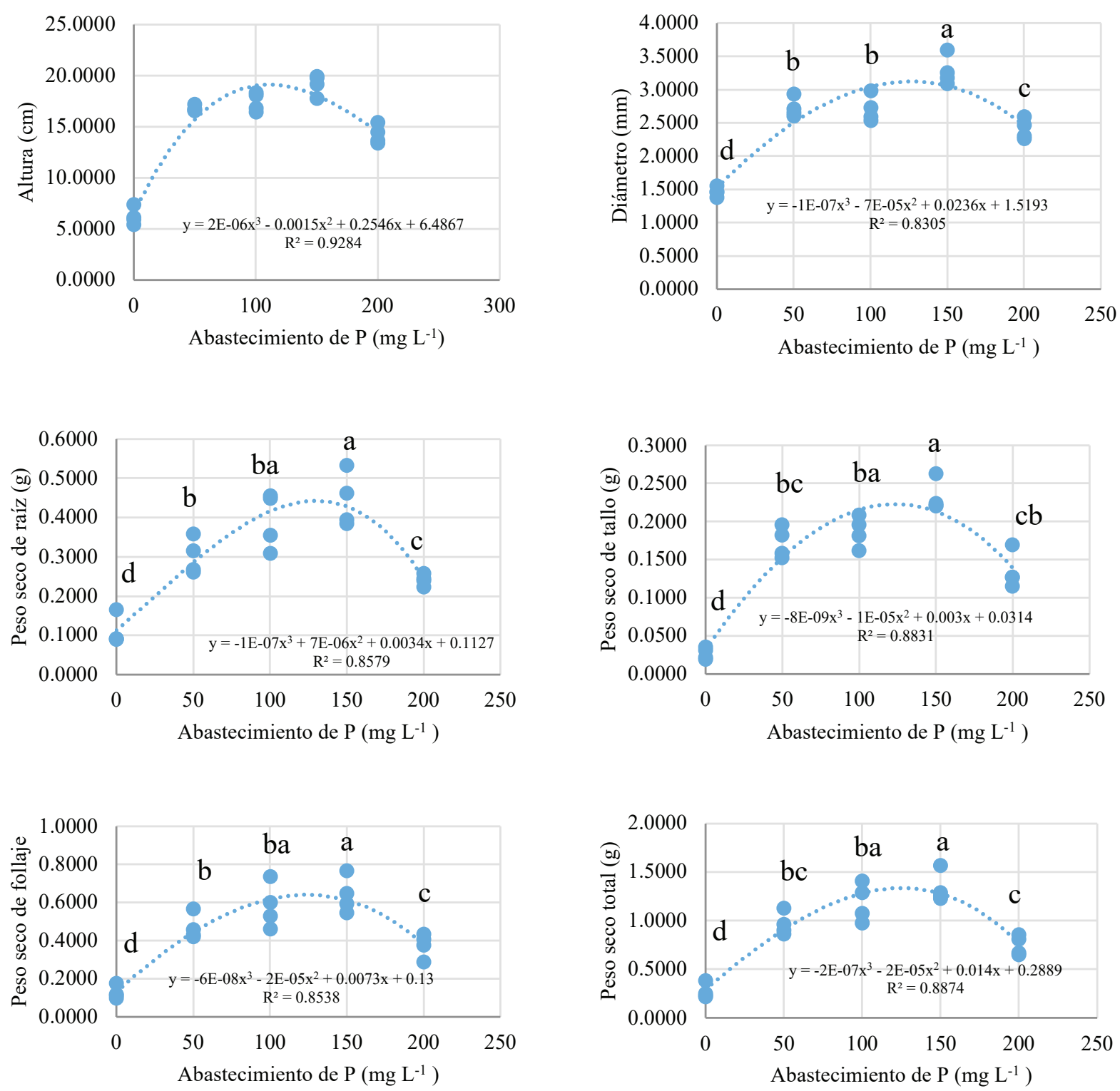

Figura 6. Curvas de abastecimiento de fósforo (P) para Pinus patula en etapa de vivero en un sistema hidropónico por subirrigación.

Figure 6. Phosphorus (P) supply curves for Pinus patula at the nursery stage, grown in a subirrigation hydroponics system.

nutritiva optimizó el crecimiento de Austrocedrus chilensis. Estos autores mencionan que la concentración de $\mathrm{K}$ en la solución nutritiva está, entre otros factores, en función de la disponibilidad de otros nutrimentos.

Como se comentó anteriormente, el presente estudio se llevó a cabo en condiciones de invernadero, mismo que se encuentra dentro de un bosque de pino, por lo que probablemente presenta limitaciones de luz, ya que se ha observado que aproximadamente a las $15: 30 \mathrm{~h}$, entre 70 y $80 \%$ de la superficie del invernadero presenta sombra de los árboles cercanos.

Bajo las condiciones mencionadas, es probable que el crecimiento de las plantas haya sido limitado por escasez de radiación solar, en cuyo caso, los requerimientos nutrimentales debieron ser menores que los que la especie tiene en condiciones óptimas de radiación solar. Ramírez $\left(2021^{2}\right)$ muestra que la altura media de planta de Pinus patula de siete meses

${ }^{2}$ Ramírez G., K. (2021). Prototipo para la producción de especies forestales en vivero mediante un sistema hidropónico por subirrigación. Tesis de Maestría en Ciencias. Postgrado en Ciencias Forestales. Colegio de Postgraduados. Texcoco, Estado de México. 65 p. 
Cuadro 4. Modificaciones propuestas a la concentración de fósforo (P) en la solución nutritiva de Hewitt y Smith, para optimizar el crecimiento de diversas variables morfológicas de Pinus patula en etapa de vivero.

Table 4. Suggested changes to the phosphorus (P) concentration in the Hewitt and Smith nutrient solution for optimizing growth of several morphological variables in Pinus patula seedlings during the nursery stage.

\begin{tabular}{|c|c|c|c|}
\hline $\begin{array}{l}\text { Variable } \\
\text { morfológica }\end{array}$ & Ecuación de abastecimiento & Primera derivada & $\mathrm{COSP}^{\dagger}$ \\
\hline Alt (cm) & $\mathrm{Alt}=2 \mathrm{E}-06 \mathrm{P}^{3}-0.0015 \mathrm{P}^{2}+0.2546 \mathrm{P}+6.4867$ & $0.000006 \mathrm{P}^{2}-0.003 \mathrm{P}+0.2546$ & 108.3 \\
\hline $\mathrm{D}(\mathrm{mm})$ & $\mathrm{D}=-1 \mathrm{E}-07 \mathrm{P}^{3}-7 \mathrm{E}-05 \mathrm{P}^{2}+0.0236 \mathrm{P}+1.51$ & $0.0000003 \mathrm{P}^{2}-0.00014 \mathrm{P}+0.0236$ & 131.5 \\
\hline PSFo (g) & $P S F o=-6 E-08 P^{3}-2 E-05 P^{2}+0.0073 P+0.13$ & $-0.00000018 \mathrm{P}^{2}-0.00004 \mathrm{P}+0.0073$ & 118.9 \\
\hline $\operatorname{PSTa}(\mathrm{g})$ & $\mathrm{PSTa}=-8 \mathrm{E}-09 \mathrm{P}^{3}-1 \mathrm{E}-05 \mathrm{P}^{2}+0.003 \mathrm{P}+0.0314$ & $-0.000000024 \mathrm{P}^{2}-0.00002 \mathrm{P}+0.003$ & 129.8 \\
\hline $\operatorname{PSRa}(g)$ & $\mathrm{PSRa}=-1 \mathrm{E}-07 \mathrm{P}^{3}+7 \mathrm{E}-06 \mathrm{P}^{2}+0.0034 \mathrm{P}+0.1127$ & $-0.0000003 \mathrm{P}^{2}+0.000014 \mathrm{P}+0.0034$ & 132.3 \\
\hline PSTo (g) & $\mathrm{PSTo}=-2 \mathrm{E}-07 \mathrm{P}^{3}-2 \mathrm{E}-05 \mathrm{P}^{2}+0.014 \mathrm{P}+0.2889$ & $-0.0000006 \mathrm{P}^{2}-0.00004 \mathrm{P}+0.014$ & 123.0 \\
\hline Media & & & 124.0 \\
\hline
\end{tabular}

Alt $=$ altura de planta; $\mathrm{D}=$ diámetro al cuello de la raíz; PSFo = peso seco de follaje; PSTa = peso seco de tallo; PSRa = peso seco de raíz; PSTo = peso seco total; $\mathrm{COSP}=$ concentración óptima de fósforo en la solución nutritiva $\left(\mathrm{mg} \mathrm{L}^{-1}\right) .{ }^{\dagger}$ Los nutrimentos restantes mantienen la concentración indicada por Hewitt y Smith. Alt = plant height; $\mathrm{D}=$ root collar diameter; PSFo = dry weight of foliage; PSTa = stem dry weight; PSRa = root dry weight; PSTo = total dry weight; COSP = optimal concentration of phosphorus in the nutrient solution $\left(\mathrm{mg} \mathrm{L}^{-1}\right) .{ }^{\dagger}$ The remaining nutrients stay at concentration indicated by Hewitt and Smith formula.

de edad, creciendo en invernadero semiabierto en la misma zona geográfica en la que se desarrolló en el presente estudio, bajo un sistema hidropónico de subirrigación, fue de $43 \mathrm{~cm}$ en un período de siete meses; en contraste, la planta correspondiente al presente experimento presentó una altura media de $19.1 \mathrm{~cm}$, en un periodo de tratamiento de cinco meses. Esto demuestra que el crecimiento de la planta en el

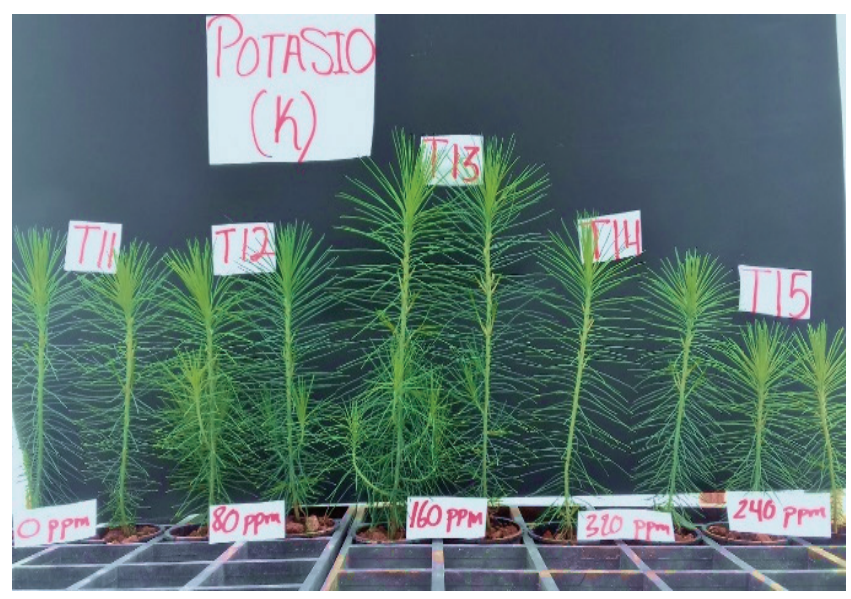

Figura 7. Comportamiento del crecimiento de altura de planta de Pinus patula en soluciones nutritivas con concentraciones crecientes de potasio (K).

Figure 7. Behavior of height growth of Pinus patula seedlings grown in nutrient solutions with increasing concentrations of potassium (K). presente estudio fue limitado por la radiación solar, puesto que el invernadero del estudio de Ramírez $\left(2021^{2}\right)$ no fue afectado por sombras de árboles.

Elliott y White (1994) señalan que cuando las plantas están limitadas por luz, la eficiencia en el uso de $\mathrm{N}$ o de $\mathrm{P}$ disminuye. Esto significa que las plantas, en esas condiciones, presentan menores necesidades nutrimentales que en condiciones normales y que el abastecimiento proporcionado probablemente ha conducido hacia un consumo de lujo por parte de las plantas, disminuyendo la eficiencia en el uso de los nutrimentos. Bigg y Schalau(1990), por su parte, señalan que la determinación exitosa de las concentraciones críticas depende de que todos los factores que afectan el crecimiento se encuentren en sus niveles óptimos; sin embargo, tal condición es extremadamente difícil de conseguir en condiciones semicontroladas como las de invernadero, por lo que resulta útil, determinar las concentraciones críticas para la especie en estudio, bajo diversas condiciones ambientales.

\section{Concentraciones Críticas}

En el Cuadro 6, se presenta un resumen de las concentraciones críticas foliares de $\mathrm{N}, \mathrm{P}, \mathrm{K}, \mathrm{Ca}, \mathrm{Mg}$, $\mathrm{Fe}, \mathrm{Cu}, \mathrm{Zn}, \mathrm{Mn}$ y B, para plántulas de Pinus patula. El mencionado cuadro, indica que la concentración crítica para un nutrimento difiere según la variable a optimizar. 

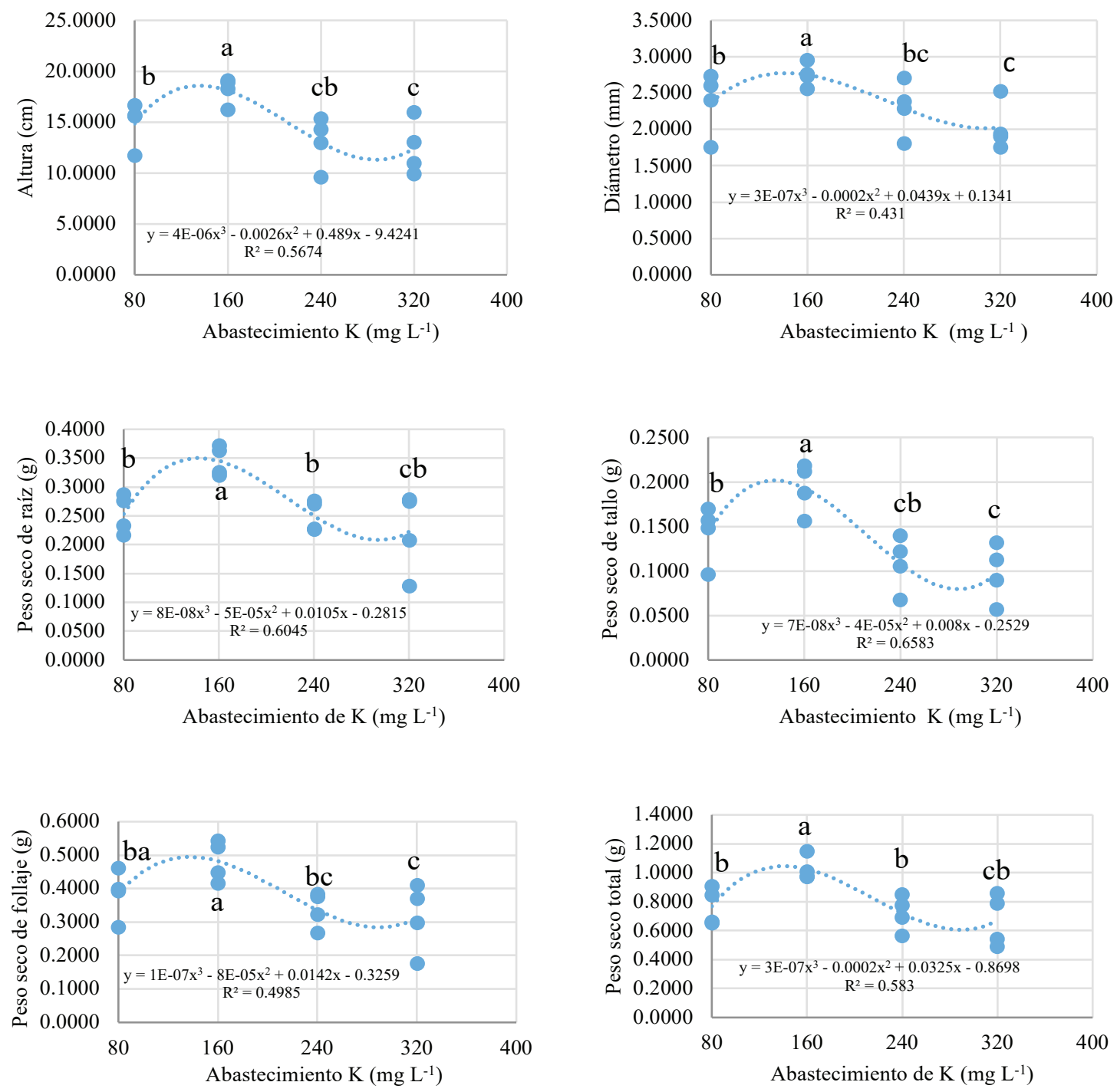

Figura 8. Curvas de abastecimiento de potasio (K) para Pinus patula en etapa de vivero en un sistema hidropónico por subirrigación.

Figure 8. Potassium (K) supply curves for Pinus patula at the nursery stage, grown in a subirrigation hydroponics system.

Esto evidencia la capacidad del manejo nutrimental en viveros, para moldear los diversos rasgos que deba tener la planta, para sobrevivir en el sitio definitivo en campo (Cortina et al., 2013). En efecto, en el cuadro se observa que, por ejemplo, si se desea privilegiar el crecimiento de follaje, se debe aumentar el abasto de $\mathrm{N}$ y $\mathrm{P}$; sin embargo, en todo caso, debe considerarse lo mencionado por Zas y Serrada (2003), en el sentido de que el nitrógeno, cuando está en exceso, puede condicionar la absorción de otros nutrimentos. Sí, por el contrario, importa tener una plántula con una elevada proporción raíz/parte aérea, entonces deberá aumentarse el abastecimiento de $\mathrm{K}, \mathrm{Fe}, \mathrm{Cu}$ y $\mathrm{Zn}$.

Solo existe un estudio publicado sobre concentraciones críticas de N, P y K en Pinus patula en etapa de vivero (Hernández y Torres, 2009³).

\footnotetext{
${ }^{3}$ Hernández Pérez, F., \& Torres L., S. (2009). Niveles críticos nutrimentales preliminares para especies forestales de México a nivel de vivero. Tesis profesional licenciatura. DICIFO, Universidad Autónoma Chapingo. Chapingo, Texcoco, México. 151 p.
} 
Cuadro 5. Modificaciones propuestas a la concentración de potasio en la solución nutritiva de Hewitt y Smith, para optimizar el crecimiento de diversas variables morfológicas de Pinus patula en etapa de vivero.

Table 5. Suggested changes to the potassium concentration in the Hewitt and Smith nutrient solution for optimizing growth of several morphological variables in Pinus patula seedlings during the nursery stage.

\begin{tabular}{|c|c|c|c|}
\hline $\begin{array}{l}\text { Variable } \\
\text { morfológica }\end{array}$ & Ecuación de abastecimiento & Primera derivada & $\operatorname{COSK}^{\dagger}$ \\
\hline Alt $(\mathrm{cm})$ & $\mathrm{Alt}=4 \mathrm{E}-06 \mathrm{~K}^{3}-0.0026 \mathrm{~K}^{2}+0.489 \mathrm{~K}-9.4241$ & $0.000012 \mathrm{~K}^{2}-0.0052 \mathrm{~K}+0.489$ & 137.9 \\
\hline $\mathrm{D}(\mathrm{mm})$ & $\mathrm{D}=3 \mathrm{E}-07 \mathrm{~K}^{3}-0.0002 \mathrm{~K}^{2}+0.0439 \mathrm{~K}+0.1341$ & $0.0000009 \mathrm{~K}^{2}-0.0004 \mathrm{~K}+0.0439$ & 162.7 \\
\hline PSFo $(g)$ & $\mathrm{PSFo}=1 \mathrm{E}-07 \mathrm{~K}^{3}-8 \mathrm{E}-05 \mathrm{~K}^{2}+0.0142 \mathrm{~K}-0.3259$ & $0.0000003 \mathrm{~K}^{2}-0.00016 \mathrm{~K}+0.0142$ & 112.5 \\
\hline $\operatorname{PSTa}(g)$ & $\mathrm{PSTa}=7 \mathrm{E}-08 \mathrm{~K}^{3}-4 \mathrm{E}-05 \mathrm{~K}^{2}+0.008 \mathrm{~K}-0.2529$ & $0.00000021 \mathrm{~K}^{2}-0.00008 \mathrm{~K}+0.008$ & 147.9 \\
\hline $\operatorname{PSRa}(g)$ & $\mathrm{PSRa}=8 \mathrm{E}-08 \mathrm{~K}^{3}-5 \mathrm{E}-05 \mathrm{~K}^{2}+0.0105 \mathrm{~K}-0.2815$ & $0.00000024 \mathrm{~K}^{2}-0.0001 \mathrm{~K}+0.0105$ & 189.7 \\
\hline PSTo $(g)$ & $\mathrm{PSTo}=3 \mathrm{E}-07 \mathrm{~K}^{3}-0.0002 \mathrm{~K}^{2}+0.0325 \mathrm{~K}-0.8698$ & $0.0000009 \mathrm{~K}^{2}-0.0004 \mathrm{~K}+0.0325$ & 107.0 \\
\hline Media & & & 143.0 \\
\hline
\end{tabular}

Estos autores seleccionaron plántulas de esta especie en varios viveros forestales de México, para determinar las concentraciones foliares y características morfológicas de aquellas que presentaron mayor crecimiento. En el estudio mencionado se concluyó que las concentraciones críticas para esta especie fueron N (0.91\%), P (0.09\%), $\mathrm{K}(0.18 \%), \mathrm{Ca}(0.15 \%), \mathrm{Mg}(0.05 \%), \mathrm{Fe}\left(17.63 \mathrm{mg} \mathrm{L}^{-1}\right), \mathrm{Cu}$ (5.83 $\left.\mathrm{mg} \mathrm{kg}^{-1}\right), \mathrm{Zn}\left(20.48 \mathrm{mg} \mathrm{kg}^{-1}\right)$ y $\mathrm{Mn}\left(93.50 \mathrm{mg} \mathrm{kg}^{-1}\right)$.
En comparación con esas concentraciones críticas, las encontradas en el presente estudio fueron elevadas, con excepción de $\mathrm{Cu}$ y $\mathrm{Zn}$, determinándose las siguientes concentraciones críticas: $\mathrm{N}(2.27 \%)$, P $(0.276 \%)$, $\mathrm{K}(0.489 \%), \mathrm{Ca}(0.329 \%), \mathrm{Mg}(0.098 \%), \mathrm{Fe}\left(86 \mathrm{mg} \mathrm{kg}^{-1}\right)$, $\mathrm{Cu}\left(4.14 \mathrm{mg} \mathrm{kg}^{-1}\right), \mathrm{Zn}\left(13.7 \mathrm{mg} \mathrm{kg}^{-1}\right)$ y Mn (437 mg kg-1). Es probable que, por un lado, las plántulas muestra de Hernández y Torres $\left(2009^{3}\right)$ hayan tenido un

Cuadro 6. Concentraciones críticas nutrimentales para Pinus patula en etapa de vivero, que optimizan diversas variables morfológicas de la planta.

Table 6. Critical nutrient concentrations for nursery-stage Pinus patula seedlings, for optimizing several morphological variables.

\begin{tabular}{|c|c|c|c|c|c|c|c|c|c|c|}
\hline Variable & $\mathrm{N}$ & $\mathrm{P}$ & K & $\mathrm{Ca}$ & $\mathrm{Mg}$ & $\mathrm{Fe}$ & $\mathrm{Cu}$ & $\mathrm{Zn}$ & $\mathrm{Mn}$ & B \\
\hline & $\%$ & $\ldots$ & $\ldots$ & $\ldots$ & $-\ldots$ & $\mathrm{ng} \mathrm{kg}$ & $-\cdots$ & $\ldots$ & $-\ldots$ & $\ldots$ \\
\hline Alt $(\mathrm{cm})$ & 1.79 & 2809 & 4881 & 3868 & 980 & 88 & 4.17 & 12.5 & 511 & 80 \\
\hline $\mathrm{D}(\mathrm{mm})$ & 2.39 & 2829 & 4862 & 3060 & 997 & 84 & 3.92 & 13.8 & 424 & 77 \\
\hline PSFo (g) & 2.46 & 2931 & 4675 & 2982 & 966 & 81 & 3.68 & 14.0 & 386 & 53 \\
\hline PSTa (g) & 2.40 & 2971 & 4680 & 3346 & 981 & 80 & 4.01 & 14.8 & 429 & 65 \\
\hline PSRa (g) & 2.23 & 2687 & 4605 & 3371 & 998 & 87 & 3.74 & 11.4 & 421 & 55 \\
\hline PSTo (g) & 2.43 & 2816 & 4522 & 2979 & 955 & 77 & 3.72 & 13.9 & 398 & 53 \\
\hline $\mathrm{R} / \mathrm{A}$ & 2.21 & 2292 & 6000 & 3405 & 988 & 106 & 5.73 & 15.5 & 493 & 51 \\
\hline Media & 2.273 & 2762 & 4889 & 3287 & 981 & 86 & 4.14 & 13.7 & 437 & 62 \\
\hline
\end{tabular}

$\overline{\mathrm{Alt}}=$ altura de planta; $\mathrm{D}=$ diámetro al cuello de la raíz; PSFo = peso seco de follaje; PSTa = peso seco de tallo; PSRa = peso seco de raíz; PSTo = peso seco total. Alt = plant height; $\mathrm{D}=$ root collar diameter; PSFo = dry weight of foliage; PSTa = stem dry weight; PSRa = root dry weight; PSTo = total dry weight. 
abastecimiento subóptimo de nutrimentos, pues la mayoría de los viveros forestales de México carecen de un programa de verificación del estado nutrimental de la producción. Por otro lado, como ya se ha mencionado, las concentraciones críticas determinadas en el presente estudio pueden estar sobreestimadas, ya que el invernadero en el que se realizó el experimento recibió sombra de árboles cercanos al mismo desde las 15:30 h aproximadamente, lo que pudo generar un efecto de concentración de nutrimentos en los tejidos de la planta (López y Estañol, 2007). No obstante, las concentraciones críticas determinadas en el presente estudio corresponden a las mayores tasas de crecimiento de la planta, lo que valida su confiabilidad.

Will (1961) reportó una concentración crítica de N para plántulas de Pinus radiata; una pinácea de rápido crecimiento, de $1.6 \%$, la cual es parecida a la que optimizó la altura de la planta en la presente investigación, pero es muy inferior a las que optimizaron otras variables de crecimiento (Cuadro 6).

No obstante que las concentraciones críticas determinadas en el presente estudio pueden ser elevadas, la mayoría de ellas se ubican dentro de los rangos descritos por Landis et al. (2005) para coníferas cultivadas en contenedor. Las concentraciones críticas que no corresponden a las indicadas por los mencionados autores son las de $\mathrm{K}, \mathrm{Mg}$ y $\mathrm{Zn}$, que son inferiores que aquellas, mientras que la de Mn resultó superior al rango definido por Landis et al. (2005) para este nutrimento.

\section{CONCLUSIONES}

Al utilizar la solución nutritiva de Hewitt y Smith (1974), las concentraciones de nitrógeno, fósforo y potasio en la solución, que optimizan el crecimiento de plántulas de Pinus patula son: 91.7, 126.0 y $142.9 \mathrm{mg} \mathrm{L}^{-1}$, respectivamente.

Las concentraciones críticas foliares para plántulas de Pinus patula en etapa de vivero fueron: $2.273 \%$ de $\mathrm{N}$, así como $\mathrm{P}\left(2762 \mathrm{mg} \mathrm{kg}^{-1}\right), \mathrm{K}\left(4889 \mathrm{mg} \mathrm{kg}^{-1}\right)$, $\mathrm{Ca}$ (3287 mg kg-1), Mg (981 $\left.\mathrm{mg} \mathrm{kg}^{-1}\right), \mathrm{Fe}\left(86 \mathrm{mg} \mathrm{kg}^{-1}\right)$, $\mathrm{Cu}$ (4.14 mg kg-1), Zn (13.7 $\left.\mathrm{mg} \mathrm{kg}^{-1}\right), \mathrm{Mn}\left(437 \mathrm{mg} \mathrm{kg}^{-1}\right)$ y B $\left(62 \mathrm{mg} \mathrm{kg}^{-1}\right)$.

La concentración crítica de cada nutrimento varía en función de la variable morfológica que se desea priorizar.

\section{DECLARACIÓN DE ÉTICA}

No aplicable.

\section{CONSENTIMIENTO PARA PUBLICACIÓN}

No aplicable.

\section{DISPONIBILIDAD DE DATOS}

Los conjuntos de datos utilizados o analizados durante el estudio actual están disponibles del autor correspondiente a solicitud razonable.

\section{CONFLICTO DE INTERESES}

Los autores declaran que no tienen intereses en competencia.

\section{FONDOS}

Los fondos necesarios para el estudio fueron provistos por el Predio Forestal Casa Redonda, Huayacocotla, Veracruz, México.

\section{CONTRIBUCIÓN DE LOS AUTORES}

Conceptualización y Metodología: F.J.M., M.Á.L.L. y E.E.B. Análisis formal e investigación: F.J.M. y M.Á.L.L. Recursos, Revisión-edición y supervisión: M.Á.L.L. y E.E.B. Preparación del borrador original: F.J.M. Adquisición de fondos: M.Á.L.L. y E.E.B.

\section{AGRADECIMIENTOS}

Al Predio Forestal Casa Redonda, Huayacocotla, Veracruz, por todo el soporte financiero.

\section{LITERATURA CITADA}

Aldana-Varajas, R., \& Aguilera R., M. (2003). Procedimientos y cálculos básicos útiles en la operación de viveros que producen en contenedor. Documento Técnico. Guadalajara, Jalisco, México: Programa Nacional de Reforestación.

Andreau, R., Giménez, D., \& Beltrano, J. (2015). Soluciones nutritivas I en cultivos hidropónicos. En J. Beltrano \& D. O. Giménez (Coord.). Cultivo en hidroponía (pp. 73-90). Buenos Aires, Argentina: Editorial de la Universidad de La Plata. 
Bigg, W. L., \& Schalau, J. W. (1990). Mineral nutrition and the target seedling. In R. Rose, S. J. Campbell, \& T. D. Landis (Eds.). Target Seedling Symposium: Proceedings, Combined Meeting of the Western Forest Nursery Associations; 1990 August 13-17; Roseburg, Oregon. General Technical Report RM-200 (pp. 139-160). Ft. Collins, CO: U.S. Department of Agriculture, Forest Service, Rocky Mountain Forest and Range Experiment Station.

Bres, W., \& Jerzy, M. (2008). Changes of nutrient concentration in chrysanthemum leaves under influence of solar radiation. Agronomy Research, 6(2), 435-444.

Cortina, J., Vilagrosa, A., \& Trubat, R. (2013). The role of nutrients for improving seedling quality in drylands. New Forests, 44, 719-732. https://doi.org/10.1007/s11056-013-9379-3

Douglass, F. J., \& Landis, T. D. (2014). Plant nutrition and fertilization. In K. M. Wilkinson, T. D. Landis, D. L. Haase, B. F. Daley, \& R. K. Dumroese (Eds.). Tropical nursery manual: a guide to starting and operating a nursery for native and traditional plants (pp. 233-252). Agriculture Handbook 732. Washington, D.C., USA: U.S. Department of Agriculture, Forest Service.

Elliott, K. J., \& White, A. S. (1994). Effects of nitrogen, and phosphorus on red pine seedling growth and nutrient use efficiency. Forest Science, 40(1), 47-58. https://doi. org/10.1093/forestscience/40.1.47

Geist, H. J., \& Lambin, E. F. (2001). What drives tropical deforestation? A meta-analysis of proximate and underlying causes of deforestation based on sub national case study evidence (LUCC report series, 4). Louvain-la-Neuve, Belgium: CIACO Printshop.

Geist, H. J., \& Lambin, E. F. (2002). Proximate causes and underlying driving forces of tropical deforestation: Tropical forests are disappearing as the result of many pressures, both local and regional, acting in various combinations in different geographical locations. BioScience, 52(2), 143-149. https:// doi.org/10.1641/0006-3568(2002)052[0143:PCAUDF]2.0. $\mathrm{CO} ; 2$

González, M., Ríos, D., Peña-Rojas, K., García, E., Acevedo, M., Cartes, E., \& Sánchez-Olate, M. (2020). Efecto de la concentración de fósforo y calcio sobre atributos morfo-fisiológicos y potencial de crecimiento radical en plantas de Aextoxicon punctatum producidas a raíz cubierta en la etapa de endurecimiento. Bosque (Valdivia), 41(2), 137-146. http://dx.doi.org/10.4067/S0717-92002020000200137

Hewitt, E. J., \& Smith, T. A. (1974). Plant mineral nutrition. England: English Universities Press.

Landis, T. D., Haase, D. L., \& Dumroese, R. K. (2005). Plant nutrient testing and analysis in forest and conservation nurseries. In R. K. Dumroese, L. E. Riley, \& T. D. Landis (Coords.). National proceedings: Forest and Conservation Nursery Associations-2004 (pp. 76-83). Fort Collins, CO: U.S. Department of Agriculture, Forest Service, Rocky Mountain Research Station.

López-López, M. Á., \& Estañol-Botello, E. (2007). Detección de deficiencias de hierro en Pinus leiophylla a partir de los efectos de dilución y concentración nutrimental. Terra Latinoamericana, 25(1), 9-15.
Louw, J. R., \& Scholes, M. C. (2003). Foliar nutrient levels as indicators of site quality for Pinus patula in the Mpumalanga escarpment area. Southern African Forestry Journal, 197(1), 21-30 https://doi.org/10.1080/20702620.2003.10431718

Mas P., J. (2003). Guía práctica para la producción de planta en un vivero. Boletín Técnico Número 5, Volumen 1. Morelia, Michoacán, México: Comisión Forestal del Estado de Michoacán.

Parra P., L. A., López L., M. Á., Gómez G., A., \& Ordaz Ch., V. M. (2020). Concentraciones críticas nutrimentales y normas DRIS para árboles de medio turno de Cedrela odorata L. Revista Mexicana de Ciencias Forestales, 11(58), 69-91. https://doi. org/10.29298/rmcf.v11i58.594

Massone, D. S., Bartoli, C. G., \& Pastorino, M. J. (2018). Efecto de la fertilización con distintas concentraciones de nitrógeno y potasio en el crecimiento de plantines de ciprés de la cordillera (Austrocedrus chilensis) en vivero. Bosque, 39(3), 375-384. https://doi.org/10.4067/S0717-92002018000300375

Prieto R., J. A., García R., J. L., Mejía B., J. M., Huchín A., S., \& Aguilar V., J. L. (2009). Producción de planta del género Pinus en vivero en clima templado frío. Publicación Especial No. 28. México: INIFAP. 49 p.

Riechelman, B., Postma, R., Specken, J., \& De Haan, J. (2021). Critical nutrient concentrations of arable crops. Literature study on the usability of critical concentrations to diagnose nutrient deficiency and/or steer fertiliser application. Rapport 1792. N.20. Wageningen: Nutriënten Management Instituut BV.

Robles V., F., Rodríguez T., D. A., \& Villanueva M., A. (2017). Calidad de planta y supervivencia en reforestación de Pinus montezumae Lamb. Revista Mexicana de Ciencias Forestales, 8(42), 55-76.

Rodríguez Laguna, R. (2010). Manual de prácticas de viveros forestales. Pachuca, Hidalgo, México: Universidad Autónoma del Estado de Hidalgo.

Rueda S., A., Benavides S., J. D., Saenz R., J. T., Muñoz F., H. J., Prieto R., J. Á., \& Orozco G., G. (2014). Calidad de planta producida en los viveros forestales de Nayarit. Revista Mexicana de Ciencias Forestales, 5(22), 58-73.

South, D. B., Starkey, T. E., \& Enebak, S. A. (2016). Forest nursery practices in the Southern United States. Reforesta, 1, 106-146. https://doi.org/10.21750/REFOR.1.07.7

Vásquez-García, I., López L., M. Á., Ángeles P., G., Trinidad S., A., Jiménez C., M., \& Aguilar B., G. (2015). Aclareo y fertilización química en la productividad primaria neta de plantaciones de Pinus patula Schiede ex Schltdl. et Cham. Revista Mexicana de Ciencias Forestales, 6(31), 82-93.

Will, G. M. (1961). The mineral requirements of radiata pine seedlings. New Zealand Journal of Agricultural Research, 4(3-4), 309-327. https://doi.org/10.1080/00288233.1961.104 20381

Zas, R., \& Serrada, R. (2003). Foliar nutrient status and nutritional relationships of young Pinus radiata D. Don plantations in Northwest Spain. Forest Ecology and Management, 174(1-3), 167-176. https://doi.org/10.1016/S0378-1127(02)00027-0 